\title{
Spectroscopic Investigation of Fluorescence Quenching Agents. Part II: Effect of Nitromethane on the Fluorescence Emission Behavior of Thirty-six Alternant Benzenoid Polycyclic Aromatic Hydrocarbons
}

\author{
SHERYL A. TUCKER, HARDJANTI DARMODJO, WILLIAM E. ACREE, JR.,* \\ JOHN C. FETZER, and MAXIMILIAN ZANDER \\ Department of Chemistry, University of North Texas, Denton, Texas 76203-5068, U.S.A. (S.A.T., H.D., W.E.A.); Chevron \\ Research and Technology Center, Richmond, California 94802-0627, U.S.A. (J.C.F.); and Rütgerswerke AG, Kekulestrasse 30, \\ D-4620 Castrop-Rauxel, Germany (M.Z.)
}

\begin{abstract}
Nitromethane is examined as a selective quenching agent for discriminating between "alternant" versus "nonalternant" polycyclic aromatic hydrocarbons in unknown mixtures. Of the 36 benzenoid solutes studied, only dibenzo[hi,wx]heptaphene failed to obey the selective quenching rule. Also reported are new experimental fluorescence probe studies for dibenzo[de,st]pentacene, dibenzo[fg,qr]pentacene, naphtho[8,1,2hij]hexaphene, benzo[vwx]hexaphene, benzo $\left[1,2,3 \mathrm{~cd} ; 4,5,6 \mathrm{c}^{\prime} \mathrm{d}\right.$ ']diperylene, dibenzo[hi,wx]heptacene, benzo[b]triphenylene, chrysense, and anthracene dissolved in nonelectrolyte solvents of varying polarity. Benzo[vwx]hexaphene was found to exhibit probe character, as evidenced by a systematic variation in emission intensity ratio with solvent polarity.
\end{abstract}

\section{INTRODUCTION}

Identification and quantification of polycyclic aromatic hydrocarbons (PAHs) in unknown mixtures require accurate fluorescence emission intensity measurements and availability of a large spectral data file for comparing the unknown's spectrum against PAH standards. To prevent misidentification, the data file should include both polar and nonpolar solvents since electronic interactions between a solvent dipole and an excited PAH solute can lead to spectral distortions, wavelength shifts and/or intensity ratio variations, as was the case with many of the polycyclic aromatic compounds examined previously. ${ }^{1-12}$ Solvent-induced fluorescence spectral changes can be rationalized qualitatively in a relatively straightforward manner. Excitation promotes the PAH solute from a ground state of low dipole moment to one of the vibrational levels of the first electronic excited state, $S_{1 v}$, with an accompanying electron distribution in the surrounding solvent molecules. Insufficient time exists, however, for solvational-sphere molecules to physically reorient with the new PAH dipole moment. Relaxation from the vibrationally excited $S_{1 v}$ level to the excited $S_{10}$ level occurs whenever solvent molecules rotationally reorient to a more stable dipole configuration during the excited state's lifetime. Emission of the fluorescence photon returns both the PAH molecule to the ground $S_{0 v}$ state and solvational molecules to their initial electronic configuration. Subsequent rotation of solvent molecules to the ground-state dipole orientation restores

\footnotetext{
Received 20 March 1992.

* Author to whom correspondence should be sent.
}

the system to its original state. Transition probabilities and energy separations between the different energy levels vary with each solute/solvent pair, and give rise to observed intensity ratio changes and emission wavelength shifts. ${ }^{13,14}$

Mixtures of environmental/industrial importance rarely contain a single component. The majority of mixtures commonly encountered contain several isomeric pairs or structurally similar PAHs, which emit in approximately the same spectral regions. Kalman filtering and Gaussian or other curve-fitting techniques, ${ }^{15-20}$ alone or in combination with phase-resolved fluorescence spectroscopy, ${ }^{21-23}$ theoretically allow uncoupling of overlapped spectra. Such methods become less reliable, however, as the number of mixture components increases. High-performance liquid chromatographic (HPLC) separation prior to fluorimetric analysis affords a viable alternative, but again the method is extremely time-consuming whenever large numbers of isomeric compounds are present.

Utilization of selective quenching agents can significantly simplify observed spectra. On the basis of limited fluorescence measurements for perylene, dibenzo$[\mathrm{b}, \mathrm{k}] \mathrm{chrysene}$, dibenzo[h,rst]pentaphene, naphtho$[1,2 \mathrm{~b}]$ fluoranthene, indeno[ $1,2,3 \mathrm{~cd}]$ pyrene, and 10,11 (peri-naphthylene)-fluoranthene dissolved in a binary aqueous-acetonitrile mixture, Blümer and Zander ${ }^{24}$ noted that nitrobenzene and nitromethane selectively quenched fluorescence emission of only the so-called "alternant" polycyclic aromatic hydrocarbons. Emission intensities of the three nonalternant PAHs (e.g., naphtho[ $1,2 \mathrm{~b}]$ fluoranthene, indeno[ $1,2,3 \mathrm{~cd}]$ pyrene, and 10,11-(peri-naphthylene)-fluoranthene) were unaffected. Although several follow-up papers ${ }^{12,25-28}$ have appeared in the chemical literature, the number of PAHs actually studied is still quite small. A complete systematic investigation needs to be undertaken in order to ascertain which alternant PAHs (if any) are exceptions to the selective quenching rule. For this reason, we report the effect that nitromethane has on the fluorescence emission of 36 PAH 6 benzenoids. These aromatic compounds (listed in Table I) are classified as alternant PAHs because every alternant carbon atom in the aromatic ring system can be "starred." Nonalternant PAHs, on the other hand, would have at least one pair of adjacent starred atoms. ${ }^{29,30}$ 
TABLE I. Summary of excitation wavelengths, probe character, and fluorescence quenching data for alternant PAH6 benzenoids.

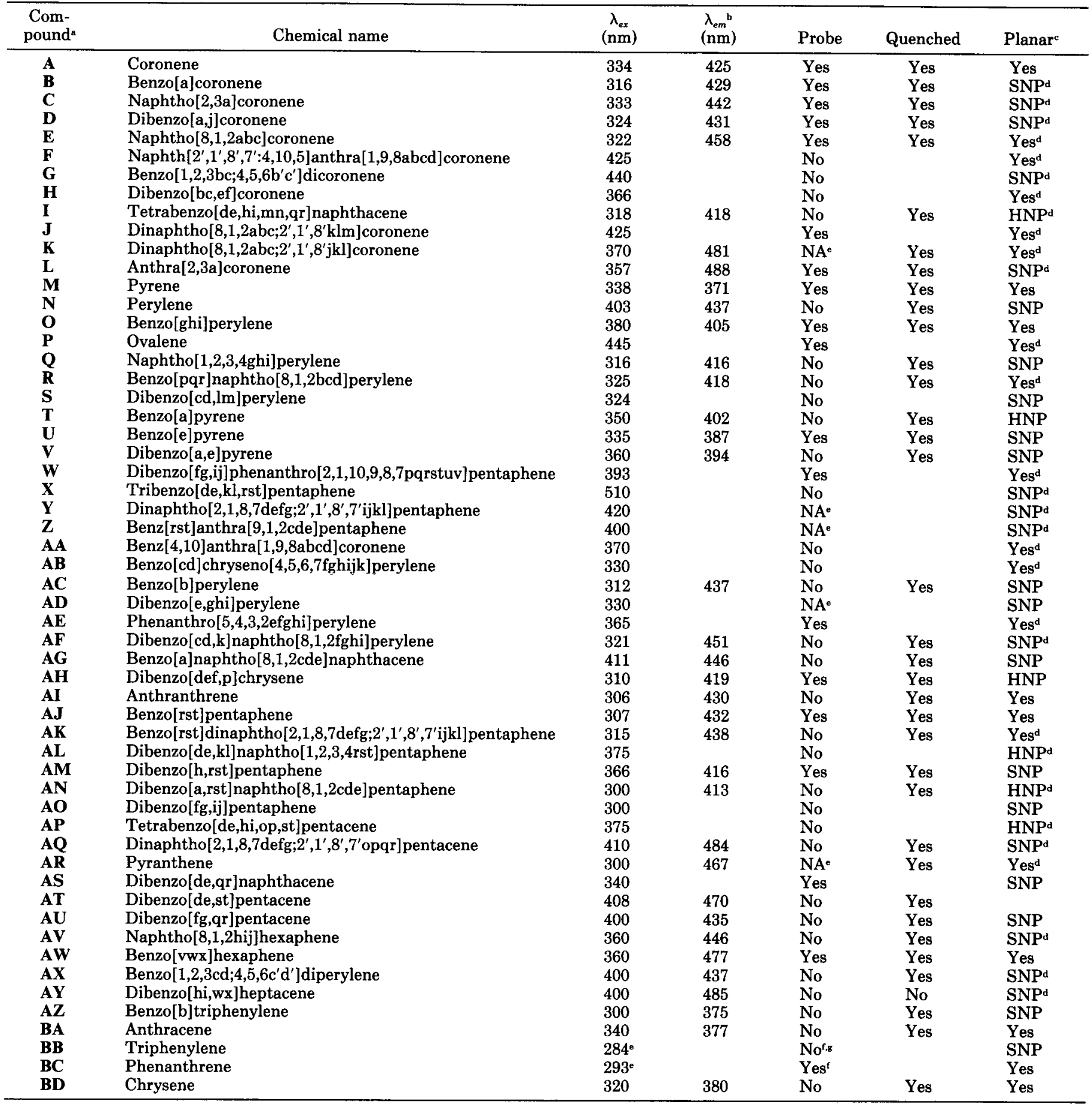

Molecular structures are given in Ref. 8.

${ }^{b}$ Emission wavelength used in nitromethane quenching studies.

c Unless otherwise noted, conformation based upon molecular mechanic computations performed by Herndon et al. ${ }^{55}$ using PCMODEL software package. Abbreviations used: HNP is highly nonplanar and SNP is slightly nonplanar.

d Predicted geometries based upon the absence/presence of sterically hindered bay regions.

- NA indicates that the PAH is unacceptable as a solvent polarity probe molecule because a common set of bands could not be assigned in all solvents studied. The molecule may exhibit selective enhancement of one (or more) fluorescence emission band(s).

$f$ Taken from the fluorescence study of Meyerhoffer and McGown. ${ }^{41}$

* Based upon the fluorescence emission spectral data reported by Nakajima.42

Also included are new experimental fluorescence probe studies for dibenzo[de,st]pentacene, dibenzo[fg,qr]pentacene, naphtho[8,1,2hij]hexaphene, benzo[vwx]hexaphene, benzo[1,2,3cd;4,5,6 $\left.\mathrm{c}^{\prime} \mathrm{d}^{\prime}\right]$ diperylene, dibenzo- [hi,wx]heptacene, benzo[b]triphenylene, chrysene, and anthracene dissolved in nonelectrolyte organic solvents of varying polarity. Molecular structures of these latter eight solutes, along with triphenylene (structure BB) and 

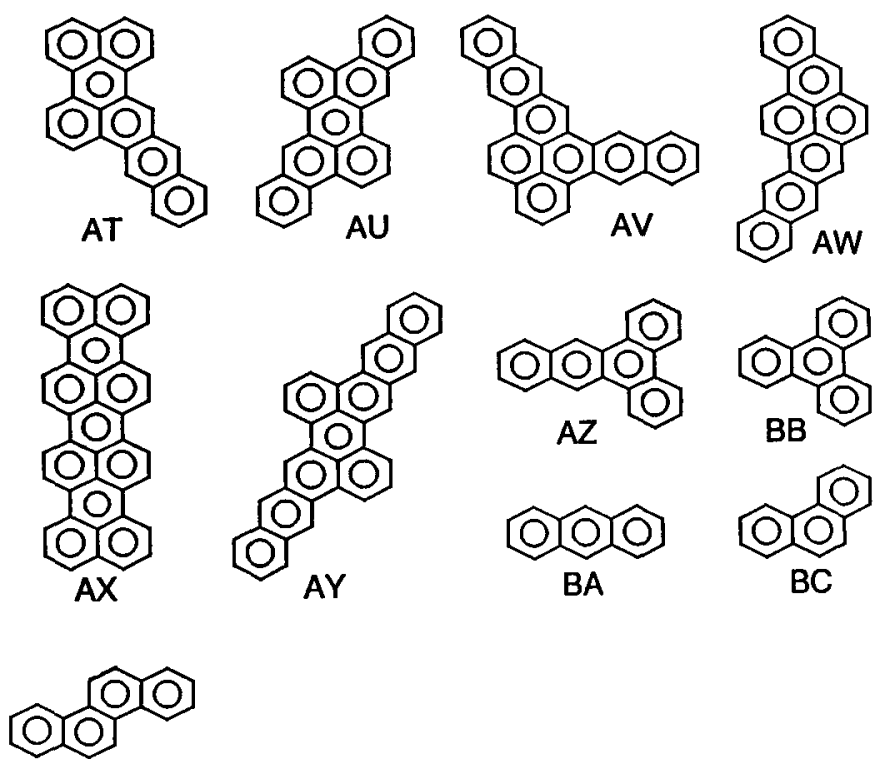

BD

FIG. 1. Molecular structures of polycyclic aromatic hydrocarbon solutes: (AT) dibenzo[de,st]pentacene; (AU) dibenzo[fg,qr]pentacene; (AV) naphtho[8,1,2hij]hexaphene; (AW) benzo[vwx]hexaphene; (AX) benzo[1,2,3cd;4,5,6c'd']diperylene; (AY) dibenzo[hi,wx]heptacene; (AZ) benzo[b]triphenylene; (BA) anthracene; (BB) triphenylene; $(\mathbf{B C})$ phenanthrene; and (BD) chrysene.

phenanthrene (BC), are depicted in Fig. 1. Structures for the remaining PAHs are given in our earlier publications $\mathrm{s}^{4,8}$ and are not repeated here.

\section{MATERIALS AND METHODS}

Anthracene ( $+99.9 \%$, Aldrich Chemical Company) and benzo[b]triphenylene (99\%, Aldrich) were purchased from commercial sources and used as received. Chrysene (98\%, Aldrich) was recrystallized several times from absolute ethanol. Dibenzo[de,st]pentacene, dibenzo[fg,qr ]pentacene, naphtho[ $[8,1,2 \mathrm{hij}]$ hexaphene, benzo[vwx] hexaphene, benzo[1,2,3cd;4,5,6 $\left.\mathrm{c}^{\prime} \mathrm{d}^{\prime}\right]$ diperylene, and dibenzo[hi,wx]heptacene were synthesized and purified by procedures described in the chemical literature. ${ }^{31-36}$ Sources of the remaining PAHs are identified in our earlier papers. ${ }^{1-8,37,38}$ Stock solutions were prepared by dissolving the solutes in dichloromethane. Small aliquots of the stock solutions were transferred into test tubes, allowed to evaporate, and diluted with the solvent of interest. Final solute concentrations were sufficiently dilute to minimize inner-filtering artifacts. Solvents were of HPLC, spectroquality, or AR grade, purchased commercially from either Aldrich or Fisher Scientific, and the resulting solutions were optically dilute (absorbances $\left.\mathrm{cm}^{-1}<0.01\right)$ at all wavelengths investigated, except for the quenching study, where the nitromethane concentration was continually increased to allow examination of inner-filtering artifacts.

Absorption spectra were recorded on a Bausch and Lomb Spectronic 2000 and a Hewlett-Packard 8450A photodiode array spectrophotometer in the usual manner with a $1-\mathrm{cm}^{2}$ quartz cuvette. The fluorescence spectra were run on a Shimadzu RF-5000U spectrofluorometer with the detector set at high sensitivity. Solutions were

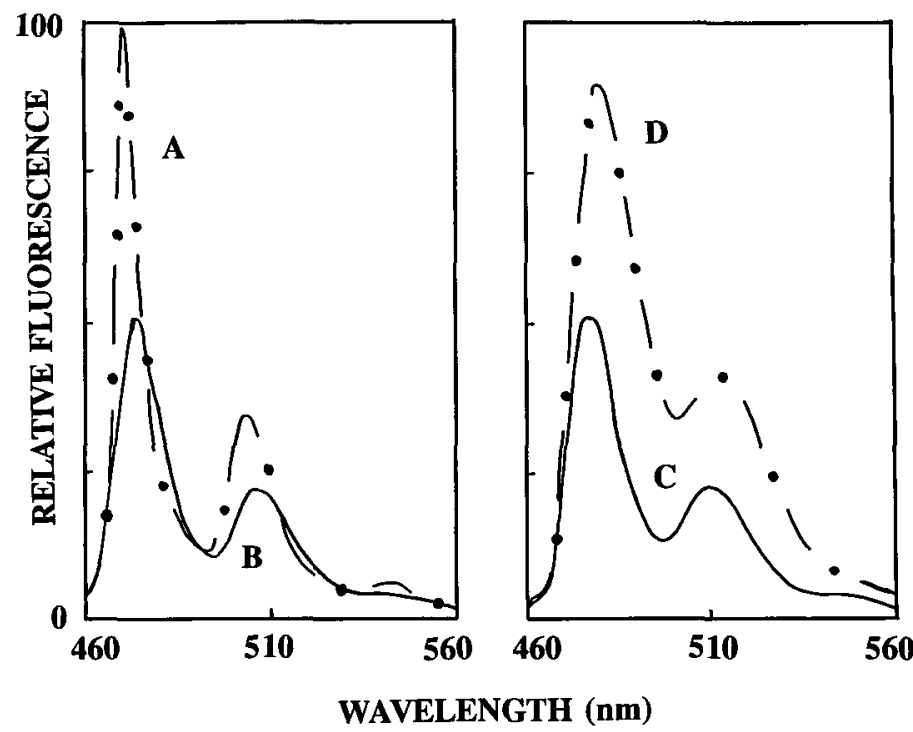

Fig. 2. Fluorescence emission spectra of benzo[vwx]hexaphene dissolved in $[A(\cdots .)]$.$n -hexadecane; [B(-)]$ butyl acetate; $[C(-)]$ dichloromethane; and $[D(\cdots-\cdot)]$ dimethyl sulfoxide. In butyl acetate major emission bands occur at about 474 and $507 \mathrm{~nm}$.

excited at the wavelengths listed in Table I. All fluorescence data were accumulated in a quartz $1-\mathrm{cm}^{2}$ cuvette at $19^{\circ} \mathrm{C}$, ambient room temperature, with excitation and emission slit width settings of $15 \mathrm{~nm}$ and $3 \mathrm{~nm}$, respectively. The PAH fluorescence spectra, depicted in Figs. $2-4$, represent a single scan which was then solvent blank corrected and verified by repetitive measurements.

\section{RESULTS AND DISCUSSION}

Representative fluorescence emission spectra of benzo[vwx] hexaphene, dibenzo[hi,wx] heptacene, and benzo[1,2,3cd;4,5,6c' $\left.\mathrm{d}^{\prime}\right]$ diperylene dissolved in $n$-hexadecane, butyl acetate, dichloromethane, and dimethyl sulfoxide are shown in Figs. 2-4. Nonelectrolyte solvents

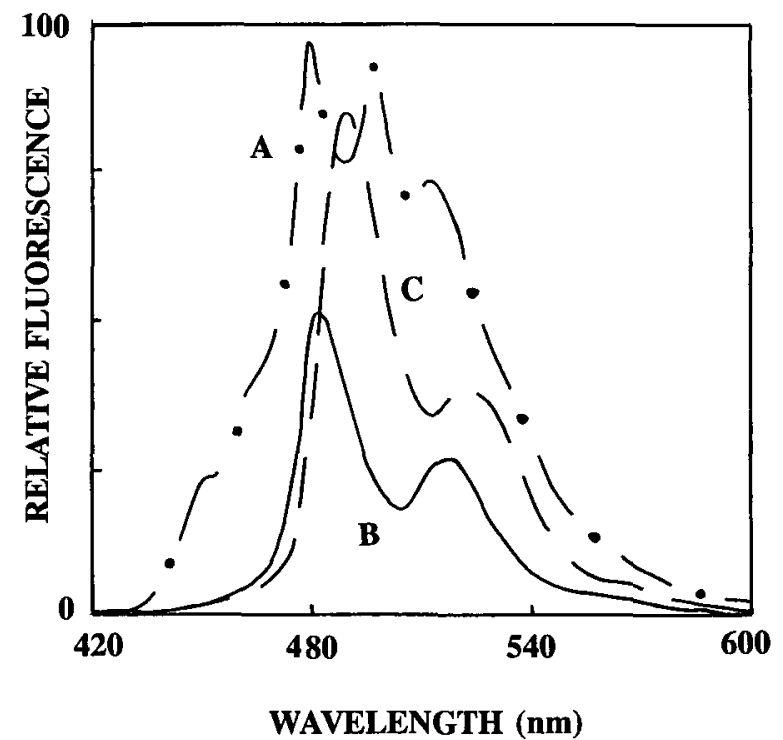

Fig. 3. Fluorescence emission spectra of dibenzo[hi,wx] heptacene dissolved in $[A(\cdots-)] n$-hexadecane; $[B(-)]$ butyl acetate; and $[C$ $(--)]$ dimethyl sulfoxide. In butyl acetate major emission bands occur at about 482 and $518 \mathrm{~nm}$. 


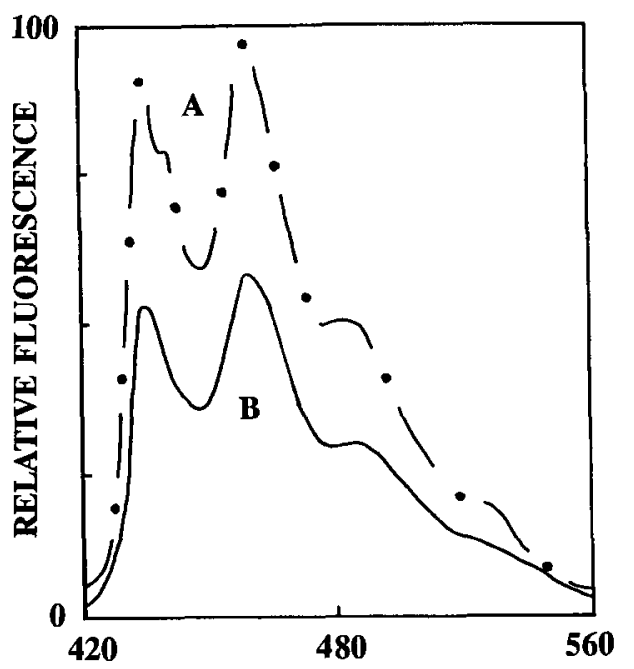

WAVELENGTH(nm)

Fig. 4. Fluorescence emission spectra of benzo[1,2,3cd;4,5,6c'd $\left.\mathrm{d}^{\prime}\right]$ diperylene dissolved in $[A(\cdots \cdots)] n$-hexadecane and $[B(-)]$ butyl acetate. In butyl acetate major emission bands occur at about 438,466 , and $496 \mathrm{~nm}$.

were judiciously selected so as to encompass the entire range of solvent polarity, from the nonpolar $n$-hexadecane hydrocarbon to the moderately polar butyl acetate and dichloromethane to the very polar dimethyl sulfoxide. Examination of the spectral data reveals that only benzo[vwx] hexaphene showed probe character, as evidenced by systematic variation of emission intensity ratios with solvent polarity. Calculated emission intensity band ratios for benzo[vwx]hexaphene (BHPh; I/II) ranged from $\mathrm{BHPh}=2.96$ for 2,2,4-trimethylpentane to $\mathrm{BHPh}=2.15$ for dimethyl sulfoxide. Actual numerical values for the 14 solvents studied are tabulated in Table II. Estimated uncertainties in the measured intensity ratios are believed to be on the order of \pm 0.08 (or less) on the basis of replicate measurements. Although ben$\mathrm{zo}[\mathrm{vwx}]$ hexaphene does show modest probe character, its respective dynamic range of 0.81 is significantly smaller than those of pyrene (1.37), benzo[ghi]perylene (0.98), and ovalene (2.08) $1,8,39$

Dibenzo[hi,wx]heptacene and dibenzo[de,st]pentacene initially appeared to possess probe character, as evidenced by a rather dramatic decrease in the I/II band emission intensity ratios in the three saturated hydrocarbon solvents. Upon closer examination it was noted that the first emission band had split into two partially resolved peaks, separated by approximately $10-15 \mathrm{~nm}$, thereby reducing the measured emission intensity. Large secondary inner-filtering artifacts, resulting from strong solute/solvent absorbance centered in the middle of an emission peak, can give rise to a "spurious hole" in the emission spectrum (see De Jersey et al. ${ }^{40}$ for a discussion of how primary inner-filtering affects excitation spectrum). This was not the case, however, in the present study. Solutions of dibenzo[hi,wx]heptacene and dibenzo[de,st]pentacene dissolved in both cyclohexane and $n$-hexadecane were optically dilute $\left(A \mathrm{~cm}^{-1}<0.01\right)$ in the 450-500 $\mathrm{nm}$ spectral region. One fewer emission band was observed in other relatively nonpolar solvents such as dibutyl ether and benzene. Calculated intensity ratios
TABLE II. Ratios of fluorescence emission intensities for benzo[vwx]hexaphene dissolved in select organic solvents.

\begin{tabular}{lcc}
\hline \multicolumn{1}{c}{ Solvent } & Py $^{\mathrm{a}}$ & $\mathrm{BHPh}^{\mathrm{b}}$ \\
\hline Cyclohexane & 0.58 & 2.90 \\
2,2,4-Trimethylpentane & 0.59 & 2.96 \\
n-Hexadecane & 0.60 & 2.94 \\
Carbon tetrachloride & & 2.77 \\
Dibutyl ether & 0.84 & 2.62 \\
Benzene & 1.05 & 2.59 \\
2-Propanol & 1.09 & 2.36 \\
Chloroform & 1.25 & 2.44 \\
Butyl acetate & 1.35 & 2.39 \\
Methanol & 1.35 & 2.27 \\
Dichloromethane & 1.35 & 2.31 \\
Acetonitrile & 1.79 & 2.15 \\
N,N-Dimethylformamide & 1.81 & 2.18 \\
Dimethyl sulfoxide & 1.95 & 2.15 \\
Dynamic Range & 1.37 & 0.81 \\
\hline
\end{tabular}

a Experimental Py values are from Dong and Winnick ${ }^{39}$ and are based upon the fluorescence emission properties of pyrene.

${ }^{b}$ Defined as the ratio of band I (at $470-480 \mathrm{~nm}$ )/II (at $502-512 \mathrm{~nm}$ ).

of $\mathrm{I} / \mathrm{II}=1.95$ and $\mathrm{I} / \mathrm{II}=2.12$ for dibenzo[hi,wx] heptacene dissolved in dibutyl ether and benzene, respectively, were essentially identical to a value of $\mathrm{I} / \mathrm{II}=2.12$ observed in dimethyl sulfoxide. Emission intensity ratios for anthracene, benzo[b]triphenylene, dibenzo[fg,qr]pentacene, naphtho[ $8,1,2 \mathrm{hij}]$ hexaphene, chrysene, and benzo$\left[1,2,3 c d ; 4,5,6 c^{\prime} d^{\prime}\right]$ diperylene either remained essentially constant or did not vary systematically with solvent polarity. Of the eight polycyclic aromatic hydrocarbon solutes examined, only benzo[vwx] hexaphene showed modest probe character.

Results from the present study, combined with our tentative classification of phenathrene as a very modest solvent polarity probe (dynamic range in the I/II intensity ratio of only 0.36 ) based upon measurements by Meyerhoffer and McGown, ${ }^{41}$ bring to 19 the total number of PAH6 benzenoids found to exhibit selective emission intensity enhancement. In contrast to Meyerhoffer and McGown, we will not list triphenylene as a PAH solvent polarity probe molecule. The extremely narrow dynamic range of triphenylene, ranging from I + II $/ \mathrm{III}=0.34$ in $n$-heptane to I + II/III $=0.46$ in acetonitrile, ${ }^{41,42}$ is much too small to permit accurate discrimination of solvent polarity.

Unfortunately, we are still no closer to understanding what molecular/structural features are responsible for probe behavior. Past studies ${ }^{5,8}$ have clearly documented that there is no simple, single correlation between probe character vs. number of $\pi$-electrons, or vs. position in the Dias PAH6 periodic table, ${ }^{43-45}$ or vs. molecular symmetry as reflected in the molecule's point group assignment. All symmetry considerations (i.e., point group assignments) assumed that the given $\mathrm{PAH}$ molecule was planar, which seem reasonable in the absence of direct structural information. Published $x$-ray crystallographic data on benzo[c]phenanthrene, ${ }^{46-48}$ dibenzo[c,g]phenanthrene, ${ }^{49}$ phenanthro[4,3c]phenanthrene, ${ }^{50}$ dibenzo[g,p]chrysene, ${ }^{51}$ naphtho[1,2,3,4rst]pentaphene, ${ }^{52}$ hexahelicene, and higher helicenes ${ }^{53,54}$ indicate that not all PAHs are planar. Intermolecular solute/solute crystal packing forces and/or the presence of sterically hindered bay-region fragments $\left(S_{4(\text { or higher) }}, T, 3 T\right.$; see Fig. 5 for definition of terms) in the molecule can lead to a non- 

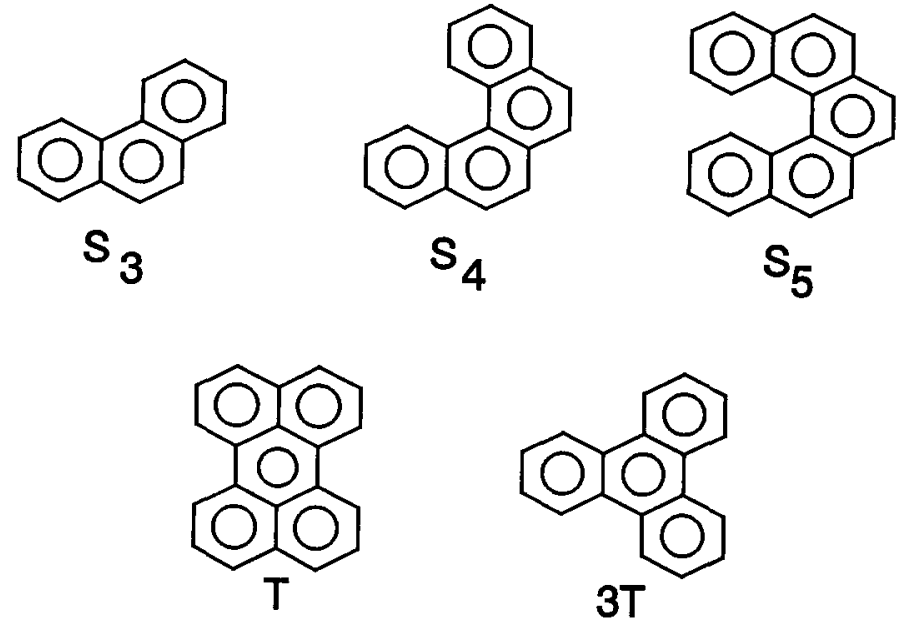

Fig. 5. Sterically hindered bay-region moieties contained in nonplanar polycyclic aromatic hydrocarbons. The $T$ parameter is characterized by condensation of phenanthroid $S_{3}$ fragments sharing a common central ring that locks the $\mathbf{S}_{3}$ bay-region hydrogen atoms in a restricted molecular environment. One $\mathbf{S}_{3} \mathbf{S}_{3}$ pair (perylene) is denoted as $T$, whereas three $\mathbf{S}_{3} \mathbf{S}_{3}$ pairs (triphenylene) equal 3T.

planar geometry. Both effects are important in the case of solid samples. Molecular structures/geometries based upon $\mathrm{x}$-ray crystallographic data may differ from those in fluid solution where the dissolved PAH is completely surrounded by solvent molecules. Recent molecular mechanic computations by Herndon et al. ${ }^{55}$ on the smaller PAH molecules containing seven phenyl-rings or less, allow us to examine possible probe character vs. planarity correlations in greater detail without imposing crystal packing constraints. Geometries of several larger PAHs were predicted on the basis of the absence/presence of sterically hindered bay regions. For example, ben$\mathrm{zo}[\mathrm{a}]$ coronene and dibenzo[a,j]coronene should be slightly nonplanar (SNP) as both molecules have $T$ bay region(s) (at least one $S_{3} S_{3}$ pair) created at the point of the added benzo-substituent ring(s). An SNP molecular geometry is consistent with that found in the corresponding pyrene-derivatives, benzo[e]pyrene and dibenzo[e,l]pyrene (formally named dibenzo[de,qr]naphthacene). Inspection of Table I reveals that 11 planar (i.e., coronene and pyrene), 1 highly nonplanar (i.e., dibenzo[def,p]chrysene), and 7 slightly nonplanar (i.e., benzo[e]pyrene and benzo[a]coronene) molecules exhibit probe character. Planarity is not a requirement for the PAH to show selective fluorescence emission intensity enhancement.

Also reported in column six of Table I are results of our investigation concerning the effect that nitromethane has on the fluorescence emission behavior of PAH6 benzenoids. As noted in the Introduction, nitromethane is reported ${ }^{24}$ to selectively quench fluorescence emission of the so-called alternant polycyclic aromatic hydrocarbons. Emission intensities of nonalternant PAHs remain unchanged for the most part; however, benzo[k]fluoranthene, fluoranthene, and naphtho[2,3b]fluoranthene are known exceptions. ${ }^{12,26,56}$ Of the alternant PAH6 benzenoids considered in the present study, only the fluorescence emission of dibenzo[hi,wx] heptacene and dibenzo[a,rst]naphtho[8,1,2cde]pentaphene (at $483 \mathrm{~nm}$ ) were not significantly diminished. The latter compound exhibited abnormal behavior in that nitromethane quenched fluorescence emission signals at 413 and 437 $\mathrm{nm}$, and only at $483 \mathrm{~nm}$ did one observe a nearly constant emission intensity. Nitromethane may undergo a chemical reaction with dibenzo[a,rst]naphtho[8,1,2cde]pentaphene, or perhaps may have changed the molecular environment around the dissolved PAH solute in such a fashion that it was also responding to solvent polarity changes. Dibenzo[a,rst]naphtho[8,1,2cde]pentaphene was originally classified as a nonprobe molecule, not because its emission intensity ratios remained constant, but rather because the observed changes did not vary systematically with solvent polarity. ${ }^{6}$

All emission intensities were corrected for primary inner-filtering artifacts arising from the absorption of excitation radiation by nitromethane. Several of the PAHs listed in Table I have excitation wavelengths in the 300$320 \mathrm{~nm}$ spectral region, and a few drops of nitromethane gave solutions having appreciable absorbances. Mathematical expressions, computational procedures, and interrogation-zone dimensions are given in our earlier papers. ${ }^{12,27}$ Secondary inner-filtering corrections were not necessary in the present study since nitromethane is "optically transparent" in these PAHs' emission ranges. Finding only one exception to the selective quenching rule in the 49 alternant PAHs examined to date, ${ }^{27}$ we believe that the addition of nitromethane to unknown PAH mixtures will provide an effective and predictable method to simplify observed fluorescence emission spectra. Additional studies are currently underway to find a reagent that selectively quenches the fluorescence emission of nonalternant polycyclic aromatic hydrocarbons. 1,2,4-Trimethoxybenzene is a promising candidate for our initial consideration. Past studies ${ }^{57,58}$ on a very limited number of compounds suggest that 1,2,4-trimethoxybenzene selectively quenches fluorescence emission of nonalternant PAHs. Further work is necessary to explore the range of applications of this preliminary observation in the analysis of multicomponent PAH mixtures.

\section{ACKNOWLEDGMENTS}

Research was supported in part by grants from the National Science Foundation (Grant No. CTS-8922485), by the University of North Texas Research Council, and by a National Science Foundation Doctoral Research Fellowship to S. A. Tucker.

1. R. Waris, M. A. Rembert, D. M. Sellers, W. E. Acree, Jr., K. W. Street, Jr., C. F. Poole, P. H. Shetty, and J. C. Fetzer, Appl. Spectrosc. 42, 1525 (1988).

2. R. Waris, M. A. Rembert, D. M. Sellers, W. E. Acree, Jr., K. W. Street, Jr., and J. C. Fetzer, Analyst 114, 195 (1989).

3. R. Waris, W. E. Acree, Jr., K. W. Street, Jr., and J. C. Fetzer, Appl. Spectrosc. 43, 845 (1989)

4. W. E. Acree, Jr., S. A. Tucker, A. I. Zvaigzne, K. W. Street, Jr., J. C. Fetzer, and H.-F. Grutzmacher, Appl. Spectrosc. 44, 477 (1990).

5. W. E. Acree, Jr., S. A. Tucker, L. E. Cretella, A. I. Zvaigzne, K. W. Street, Jr., J. C, Fetzer, K. Nakasuji, and I. Murata, Appl. Spectrosc. 44, 951 (1990).

6. S. A. Tucker, A. I. Zvaigzne, W. E. Acree, Jr., J. C. Fetzer, and M. Zander, Appl. Spectrosc. 45, 424 (1991).

7. S. A. Tucker, I.-L. Teng, W. E. Acree, Jr., and J. C. Fetzer, Appl. Spectrosc. 45, 186 (1991).

8. W. E. Acree, Jr., S. A. Tucker, and J. C. Fetzer, Polycyclic Aromat. Compds. 2, 75 (1991). 
9. S. A. Tucker, W. E. Acree, Jr., and M. J. Tanga, Appl. Spectrosc. 45, 57 (1991).

10. S. A. Tucker, W. E. Acree, Jr., and M. J. Tanga, Appl. Spectrosc. 45, 911 (1991).

11. S. A. Tucker, W. E. Acree, Jr., M. J. Tanga, M. Zander, J. C. Fetzer, S. Tokita, K. Hiruta, K. Kitahara, and H. Nishi, Appl. Spectrosc. 45, 1188 (1991).

12. S. A. Tucker, W. E. Acree, Jr., B. P. Cho, R. G. Harvey, and J. C. Fetzer, Appl. Spectrosc. 45, 1699 (1991).

13. T. L. Cecil and S. C. Rutan, Anal. Chem. 62, 1998 (1990).

14. J. R. Lakowicz, Principles of Fluorescence Spectroscopy (Plenum Press, New York, 1983).

15. R. E. Kalman, J. Basic Eng. 82, 34 (1964).

16. S. C. Rutan, J. Chemom. 1, 7 (1987).

17. S. D. Brown, Anal. Chim. Acta 181, 1 (1986).

18. S. C. Rutan, D. D. Gerow, and G. Hartmann, Intell. Lab. Sys. 3, 61 (1988).

19. H. Gampp, M. Maeder, C. J. Meyer, and A. D. Zuberbühler, Talanta 32, 1133 (1985).

20. M. Maeder, Anal. Chem. 59, 527 (1987).

21. D. W. Millican and L. B. McGown, Appl. Spectrosc. 46, 28 (1992).

22. D. W. Millican and L. B. McGown, Anal. Chem. 61, 580 (1989).

23. D. W. Millican and L. B. McGown, Anal. Chem. 62, 2242 (1990).

24. G.-P. Blümer and M. Zander, Fresenius Z. Anal. Chem. 296, 409 (1979).

25. S.-H. Chen, C. E. Evans, and V. L. McGuffin, Anal. Chim. Acta 246, 65 (1991).

26. A. F. Olea and J. K. Thomas, J. Am. Chem. Soc. 110, 4494 (1988).

27. V. L. Amszi, Y. Cordero, B. Smith, S. A. Tucker, W. E. Acree, Jr., C. Yang, E. Abu-Shaqara, and R. G. Harvey, Appl. Spectrosc. 46, 1156 (1992).

28. P. L. Konash, S. A. Wise, and W. E. May, J. Liq. Chromatogr. 4, 1339 (1981).

29. H. E. Zimmerman, Quantum Mechanics for Organic Chemists (Academic Press, New York, 1975), pp. 145-146.

30. J. March, Advanced Organic Chemistry: Reactions, Mechanisms and Structure (McGraw-Hill, New York, 1968), pp. 46-48.

31. E. Clar, C. T. Ironside, and M. Zander, Tetrahedron 22, 3527 (1966).

32. E. Clar, Berichte 65, 846 (1932).
33. E. Clar, Polycyclic Aromatic Hydrocarbons (Academic Press, New York, 1964), Vol. 2, p. 53.

34. M. Zander and W. Franke, Chem. Ber. 94, 446 (1961).

35. E. Clar and M. Zander, Tetrahedron 19, 521 (1963).

36. E. Clar, W. Kelley, and R. M. Laird, Monatshefte 87, 391 (1956).

37. J. C. Petzer and W. R. Biggs, J. Chromatogr. 295, 161 (1984).

38. J. C. Fetzer and W. R. Biggs, J. Chromatogr. 322, 275 (1985).

39. D. C. Dong and M. A. Winnick, Can. J. Chem. 62, 2560 (1984).

40. J. De Jersey, P. Jeffers-Morley, and R. B. Martin, Biophys. Chem. 13, 233 (1981).

41. S. M. Meyerhoffer and L. B. McGown, Anal. Chem. 63, 2082 (1991).

42. A. Nakajima, J. Lumin. 8, 266 (1974).

43. J. R. Dias, Acc. Chem. Res. 18, 241 (1985).

44. J. R. Dias, Handbook of Polycyclic Aromatic Hydrocarbons, Part A (Elsevier, Amsterdam, The Netherlands, 1987).

45. J. R. Dias, Handbook of Polycylic Aromatic Hydrocarbons, Part B (Elsevier, Amsterdam, The Netherlands, 1988).

46. F. H. Herbstein and G. M. J. Schmidt, J. Chem. Soc., 3302 (1954).

47. A. L. Hirshfeld, S. Sandler, and G. M. J. Schmidt, J. Chem. Soc., 2108 (1963).

48. A. L. Hirshfeld, J. Chem. Soc., 2126 (1963).

49. A. O. McIntosh, J. M. Robertson, and V. Vand, J. Chem. Soc., 1661 (1954).

50. C. De Rango, G. Tsoucaris, J. P. Declerq, G. Germain, and J. P. Petzeys, Cryst. Struct. Comm. 2, 189 (1973).

51. F. H. Herbstein, Acta Crystallogr. B35, 1661 (1979).

52. P. J. Roberts and G. Ferguson, Acta Crystallogr. B33, 1244 (1977).

53. W. H. Laarhoven and W. J. C. Prinsen, Top. Curr. Chem. 125, 63 (1984) and references therein.

54. K. P. Meurer and F. Vogtle, Top. Curr. Chem. 127, 1 (1985) and references therein.

55. W. C. Herndon, P. C. Nowak, D. A. Conner, and P. Lin, J. Am. Chem. Soc. 114, 41 (1992).

56. H. Dreeskamp, E. Koch, and M. Zander, Z. Naturforsch. 30A, 1311 (1975).

57. U. Breymann, H. Dreeskamp, E. Koch, and M. Zander, Fresenius Z. Anal. Chem. 293, 208 (1978).

58. U. Breymann, H. Dreeskamp, E. Koch, and M. Zander, Chem. Phys. Lett. 59, 68 (1978). 